\title{
The Coupled Eulerian-Lagrangian Analysis of the KOBO Extrusion Process
}

\author{
Marta Wójcik ${ }^{1 *}$, Andrzej Skrzat ${ }^{1}$ \\ 1 Department of Materials Forming and Processing, Rzeszow University of Technology, Al. Powstańców \\ Warszawy 8, 35-959 Rzeszów, Poland \\ * Corresponding author's e-mail: m.wojcik@prz.edu.pl
}

\begin{abstract}
The KOBO extrusion is an unconventional elastic-plastic deformation process in which the phenomenon of changing a path of plastic deformation due to die cyclic oscillations by a given angle and with a given frequency is applied. As the result of the application of the oscillating rotary motion of the die, the reduction of the extrusion force was obtained. The numerical study of the KOBO extrusion of metallic materials was presented in this paper. The three-dimensional coupled Eulerian-Lagrangian (CEL) analysis was applied. The relationship between the extrusion force and the punch displacement during the KOBO process was achieved. The effective plastic strain distribution in the butt was found. The results of the numerical computations were compared with the experimental data. The influence of the material hardening parameters on the cyclic loading phenomena (ratcheting, mean stress relaxation) in terms of the course of the KOBO extrusion was also examined. The proper determination of the material hardening parameters can help to optimize the KOBO process in terms of the reduction the extrusion force and the choice of the amount of die oscillations.
\end{abstract}

Keywords: coupled Eulerian-Lagrangian method, KOBO extrusion, metal forming, finite element method, hardening parameters, cyclic loading

\section{INTRODUCTION}

The extrusion technology has been more and more involved in the production of solid, semi-solid and hollow profiles due to its ability to achieve a good precision and quality, as well as homogenous properties throughout the product [1]. Extrusion is the most often used for the production of pipes, rods, profile shapes and machine elements made of, e.g. steel, as well as non-ferrous metals and their alloys. The main advantages of the extrusion are the possibility to obtain products of different shapes, which are characterized by the good mechanical properties and favorable structure as well as the occurrence of triaxial non-uniform compression stresses which determines the forming of materials with a low plasticity [2]. Additionally, the extrusion process allows obtaining high strain degrees in only single forming operation. On the other hand, in order to form hard deformable metals, the conventional extrusion process requires high extrusion forces and high temperatures. Unfortunately, the application of hot extrusion metal forming does not guarantee the preferable properties of final products. Therefore, there is a need to apply low cost extrusion methods which enable to decrease the forming forces, as well as to obtain the products with a favorable structure determining expected final properties. The $\mathrm{KOBO}$ extrusion is an unconventional elasticplastic deformation process counted among $\mathrm{Se}$ vere Plastic Deformation (SPD) methods which uses the phenomenon of changing a path of plastic deformation by introducing die cyclic oscillations by a given angle (approximately $5-7^{\circ}$ ) and with a given frequency (usually several Hertz) [3]. The application of the cyclic bilateral torsion of extruded material causes that the material evolves to the state of fluid while a solid 
state is maintained. Additionally, cyclic oscillations of the die result in the interference in the structure of a material and leads to an increased concentration of lattice defects [4].

The high yield stress and the tension strength, as well as the thermal stability are the main advantages of the products obtained with the use of the KOBO extrusion process. The maintenance of a constant rate and the constant extrusion force at the same time causes that according to the Newton's laminar flow principle (Eq. 1), a viscosity coefficient has a constant value which also determines the qualitative and quantitative invariability of the process course and determines the same properties of products on the length [5]. The Eq. 1 also confirms the linear relationship between the stress and the strain rate during the KOBO extrusion process. The application of SPD methods, including the KOBO process, leads to the hardening of material in the wake of the dislocation hardening [6].

$$
\sigma=\eta \cdot \dot{\varepsilon}
$$

where: $\sigma$-flow stress,

$\eta$ - viscosity coefficient,

$\dot{\varepsilon}-$ strain rate.

Numerical modeling of metal forming processes including the extrusion plays an important role in their optimization, leading to a decrease of forming forces and total costs. Although numerical simulations of the conventional extrusion are presented in the literature [7-10], there are very few numerical analyses considering the KOBO extrusion process. Most of them focus on the evolution of the material structure during the process. The results of modeling of a texture evolution in the KOBO extrusion are contained in [11]. The model including generation, interaction and annihilation of point defects in the KOBO process is described in [12].

Numerical simulations of the KOBO extrusion process are presented in this paper. The three-dimensional coupled Eulerian-Lagrangian approach (the CEL method) with explicit integration was applied. The results of the numerical computations were verified experimentally. It was noted that proposed model ensures good convergence between experimental and numerical results and can be used for describing the material response during the $\mathrm{KOBO}$ extrusion process. The influence of hardening characteristics on the cyclic loading phenomena in terms of the course of the KOBO process was also examined.

\section{THE COUPLED EULERIAN-LAGRANGIAN APPROACH}

In numerical simulations of large deformation problems, the Langrangian and Eulerian methods are applied to describe the movement of a small volumetric element as a function of time [13]. In the Lagrangian approach used in the solid mechanics, the movement of a continuum is determined as a function of materials coordinates and time. The nodes and elements of a Lagrangian mesh move together with a material [14] (Figure 1a). In the case of large deformations, remeshing is quite often necessary. The results of numerical simulations of the typical extrusion process are described in $[15,16]$.

The Eulerian method can be applied as an alternative to the Lagrangian approach. In the Euler method, which is applied mostly in the fluid mechanics, the movement of a continuum is a function of spatial coordinates and time [17]. In this approach, an Eulerian reference mesh remains undistorted and is used for tracking the motion of a material in the Eulerian domain $[18,19]$. The material can move through the Eulerian mesh and the distortion of elements does not occur (Figure 1b).

It is worth noting that both mentioned methods have some disadvantages. In the Lagrangian approach, as the finite element mesh become distorted under loading, the generation of completely new mesh is necessary, which causes convergence problems and extends the computational

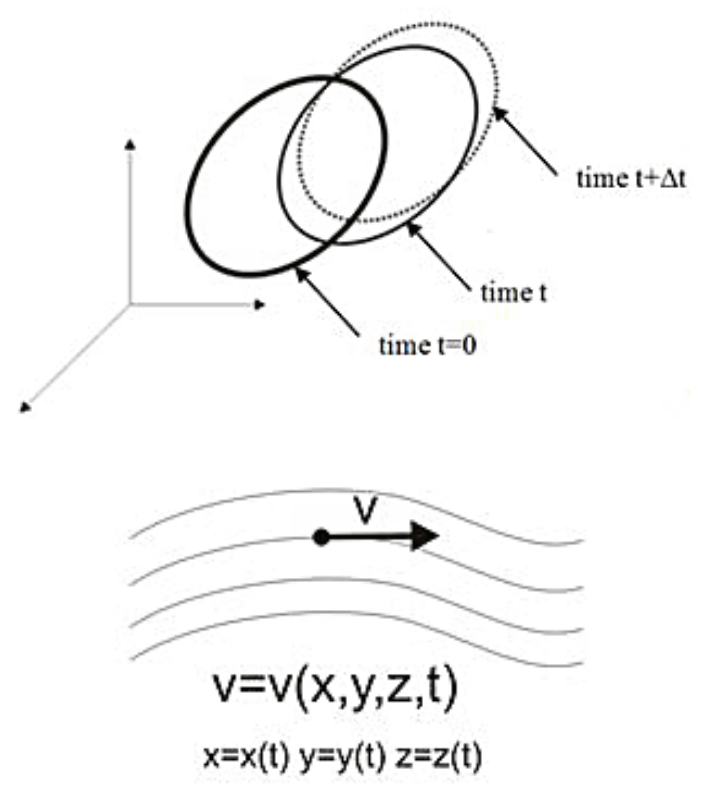

Figure 1. (a) Total and Updated Lagrangian methods; (b) the Eulerian method 
time [20]. In turn, in the Eulerian method, the material flows through the mesh without its deformation and the remeshing is not needed [21-23]. However, the Eulerian approach has some limitations e.g. material anisotropy and residual stresses are not considered.

In order to improve the convergence and reduce the time of calculations, mixed methods e.g. the Arbitrary Lagrangian-Eulerian (ALE) and Coupled Eulerian-Lagrangian (CEL) can be used. Due to the complexity of the modeling of the KOBO extrusion process, the CEL method was proposed for numerical computations. This method, which captures the advantages of both the Lagrangian and Eulerian approaches, can be used for solving fluid-structure interaction problems [10].

In the Eulerian analyses, equations are written with the use of spatial time derivatives, while material time derivatives are applied in the Lagrangian approaches in the solid and structural analyses. The relationship between material and spatial time derivatives can be written as follows [24]:

$$
\frac{D \Phi}{D t}=\frac{\partial \Phi}{\partial t}+v \cdot(\nabla \Phi)
$$

where: $\Phi$ - arbitrary solution variable,

$v$-material velocity,

$D \Phi / D t$ - material derivative,

$\partial \Phi / \partial t$ - spatial derivative.

The Lagrangian mass, momentum and energy conservation equations transferred into the Eulerian (spatial derivatives) conservation equations are (Eq. 3-5) [25]:

$$
\begin{gathered}
\frac{\partial \rho}{\partial t}+v \cdot(\nabla \rho)+\rho \nabla \cdot v=0 \\
\frac{\partial v}{\partial t}+v \cdot(\nabla \cdot \mathrm{v})=\frac{1}{\rho}(\nabla \cdot \boldsymbol{\sigma})+\boldsymbol{b} \\
\frac{\partial e}{\partial t}+v \cdot(\nabla e)=\boldsymbol{\sigma}: \boldsymbol{D}
\end{gathered}
$$

where: $\rho$-density,

$\sigma-$ Cauchy stress,

$\boldsymbol{b}-$ vector of body forces,

$e-$ strain energy,

$\boldsymbol{D}$ - velocity strain.
The mentioned Eulerian equations can be arranged into the conservative forms in the following (Eq. 6-8) [24, 25]:

$$
\begin{gathered}
\frac{\partial \rho}{\partial t}+\nabla \cdot(\rho v)=0 \\
\frac{\partial \rho v}{\partial t}+\nabla \cdot(\rho v \otimes v)=\nabla \cdot \boldsymbol{\sigma}+\rho \boldsymbol{b} \\
\frac{\partial e}{\partial t}+\nabla \cdot(e v)=\boldsymbol{\sigma}: \boldsymbol{D}
\end{gathered}
$$

The Eulerian governing equations (Eq. 6-8) can be described in the common general conservative form (Eq. 9) [24]:

$$
\frac{\partial \phi}{\partial t}+\nabla \cdot \phi=S
$$

where: $\phi$ - flux function,

$$
S \text { - source term. }
$$

The operator splitting divides the aforementioned equation (Eq. 9) into two equations solved sequentially (Eq. 10-11) [25]:

$$
\begin{gathered}
\frac{\partial \phi}{\partial t}=S \\
\frac{\partial \phi}{\partial t}+\nabla \cdot \Phi=0
\end{gathered}
$$

Eq. 10, which represents the Lagrangian step, contains the source term and the Eq. 11, describing the Eulerian step, includes the convective term.

The graphical interpretation of the split operator is shown in Figure 2. In order to solve Eq. (11), the deformed (Lagrangian) mesh is moved to the original (Eulerian) fixed mesh and the volume of material transported between adjacent elements is computed. The Lagrangian solution variables, such as the mass, energy, momentum, stress and others are then adjusted to account for the flow of the material between adjacent elements by the transport algorithms [25].

The main advantage of the CEL method is that the approach does not demand the remeshing. A unique feature of the CEL method is that a single volume can be filled simultaneously with many materials which allows simulating the extrusion of composite and porous materials. Additionally,

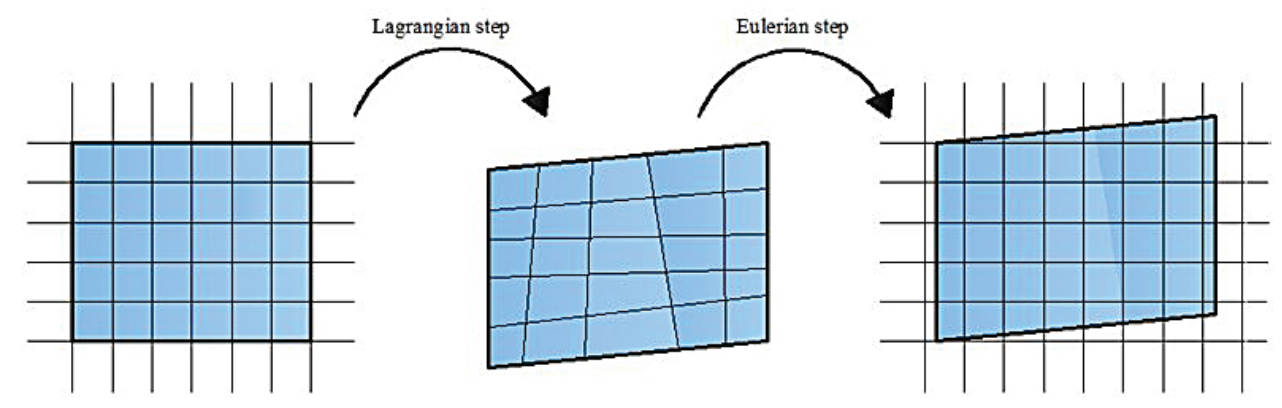

Figure 2. The graphical interpretation of a split operator in the CEL method 
this approach ensures better interpretation of contact conditions than the Eulerian method. Moreover, the classical FEM methods based on the Lagrangian approach often cause the contact problems entailed by the distortion of the mesh [20]. On the other hand, the very small stable time increment in the explicit integration procedure of approximately $10^{-6}-10^{-7} \mathrm{~s}$ is the main disadvantage of this method.

\section{THE EXPERIMENTAL PROCEDURE}

The experimental test of the KOBO extrusion process was carried out using the HYDROMET hydraulic press with the maximum load of 2.5 MN (Figure 3). The investigation was conducted on M1E copper. The properties of extruded material are presented in Table 1 . The prepared billets had the diameter of $60 \mathrm{~mm}$, corresponding to the press container dimensions.

The parameters of the KOBO extrusion process were as follows: $0.1 \mathrm{~mm} / \mathrm{s}$ ram displacement, $6 \mathrm{~Hz}$ oscillation frequency and $\pm 6^{\circ}$ swing angle from starting position. The press was cooled with water at room temperature on the exit of the press.

The macrostructure of butts obtained was examined. During the KOBO extrusion process, the maximum force was also registered. Two types of dies (Figure 4) are used in order to investigate the influence of the die notches shapes on the punch

Table 1. Properties of M1E copper used in a research

\begin{tabular}{|l|c|}
\hline \multicolumn{1}{|c|}{ Parameter } & Value \\
\hline Young modulus $[\mathrm{MPa}]$ & 130000 \\
\hline Density $\left[\mathrm{kg} / \mathrm{m}^{3}\right]$ & 8900 \\
\hline Hardness $[\mathrm{HB}]$ & 50 \\
\hline Poisson's ratio & 0.33 \\
\hline
\end{tabular}

force. These preliminary investigations are considered later on in the numerical simulations by including different contact formulations between the die and extruded material.

\section{THE FEM MODEL OF THE KOBO EXTRUSION PROCESS}

The coupled Eulerian-Lagrangian method is very useful in numerical simulations of materials forming and processing with very large deformations. The assembly of the 3-D FEM model used in the KOBO extrusion analysis is shown in Figure 5. The main data of the model are contained in Table 2. The die and the recipient are modeled as rigid bodies and the extruded material is considered as the elastic-plastic deformable body. The Eulerian part covers both the initial and the final position of the extruded material.

The simplified (flat) shape of the die was assumed. In order to distinguish two types of dies used in experiment (Figure 4), two contact formulations were considered. The first one allows material sliding on the die. In the second approach, the rough contact is assumed, which prevents from sliding between the die and extruded material. The change of the punch force can be observed for both contact formulations.

In the CEL method, the Eulerian material is tracked as it flows through the mesh by computing so-called Eulerian Volume Fraction (EVF). Each Eulerian element is designated as a percentage which shows the portion of that element filled with the material. If an Eulerian element is completely filled with the material $E V F=1$, otherwise, $\mathrm{EVF}=0$ (it can be interpreted that material is filled with "void" material) [26].

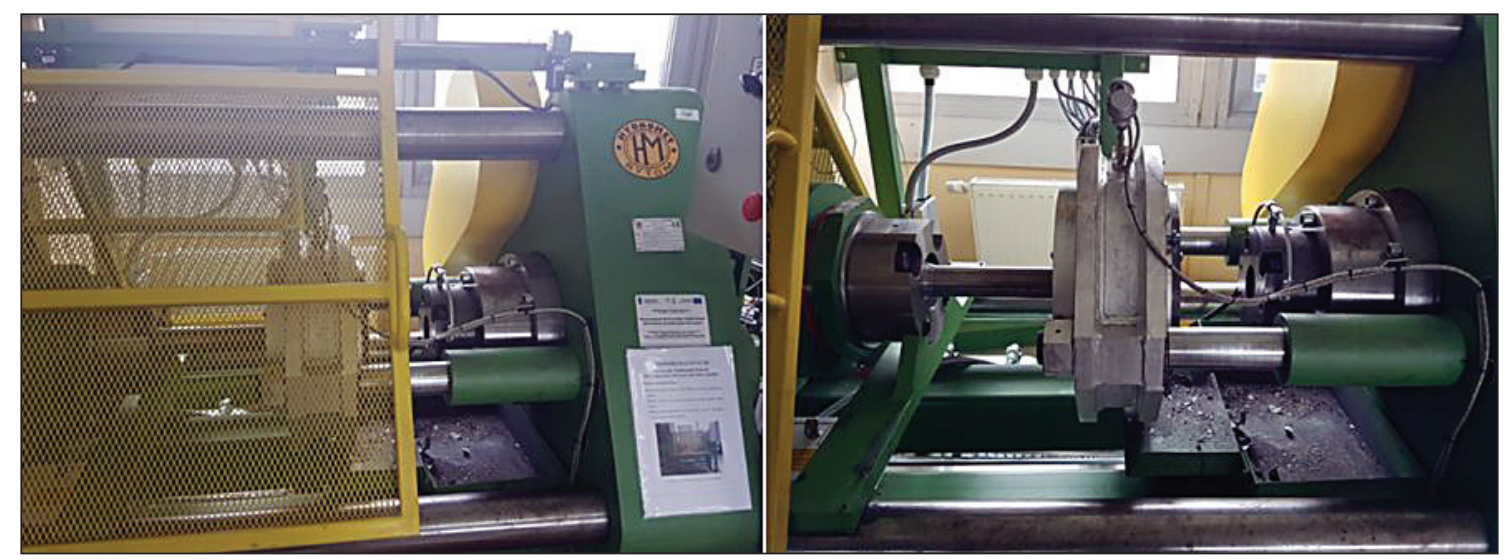

Figure 3. The KOBO hydraulic press used in a research 


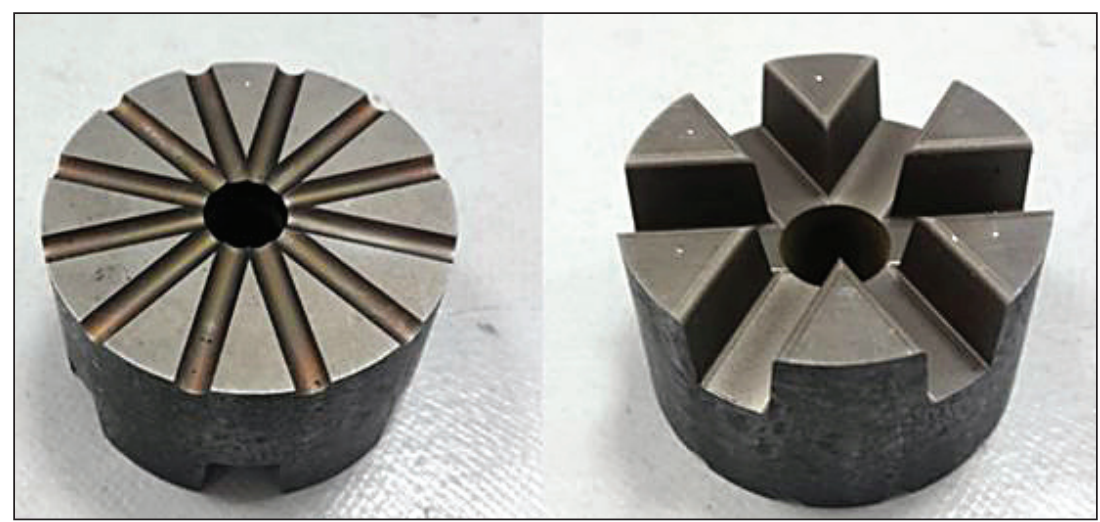

Figure 4. Different shapes of dies used in experimental investigations

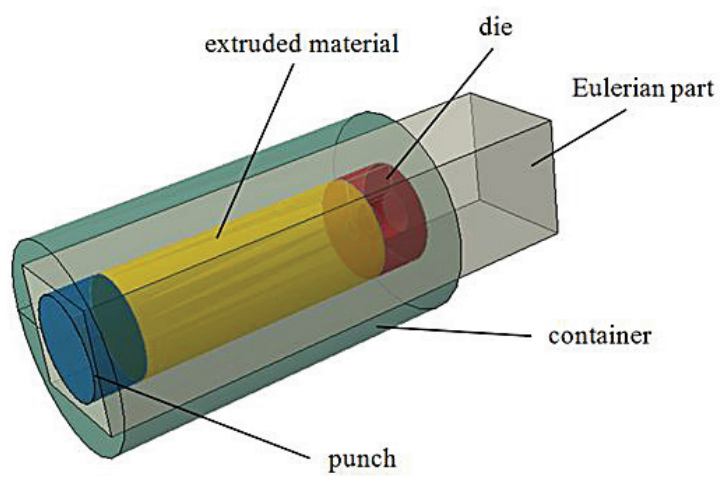

Figure 5. The FEM model of the $\mathrm{KOBO}$ extrusion process

The CEL problems are solved as an dynamic explicit analysis. The central difference rule is used for the solution of the non-linear system of differential equations [27]. The unknown solution is found directly from the results from the previous step. For each time increment, the Lagrangian phase is calculated first. Next, in the Eulerian phase, the movement and the position of the Eulerian material are determined.

The explicit procedure is conditionally stable and the stable time increment is very small i.e. $10^{-5}-10^{-7} \mathrm{~s}$. The solution of the problem usually requires hundreds of thousands time increments. In practice, the problem cannot be solved in real process time.

Some features in the ABAQUS program allow for the acceleration of computations: the mass scaling, short-time load application in the smooth step.

In numerical simulations, the time of real extrusion has been shortened by about one order. In order to avoid very large inertia forces caused by the sudden punch movement, the punch displacement from the time to was described by (Eq. 12) [24]:
Table 2. Details of the FEM model

\begin{tabular}{|l|l|}
\hline \multicolumn{1}{|c|}{ Feature } & \multicolumn{1}{c|}{ Description } \\
\hline Numerical model & 3-D \\
\hline Material model & elastic-plastic \\
\hline $\begin{array}{l}\text { Integration } \\
\text { procedure }\end{array}$ & dynamic explicit \\
\hline Friction formulation & Coulomb friction \\
\hline Friction coefficient & 0.2 \\
\hline Tool type & rigid bodies \\
\hline Steps & two steps \\
\hline Step time & $0.2 \mathrm{~s}$ \\
\hline $\begin{array}{l}\text { Number of } \\
\text { elements }\end{array}$ & $\begin{array}{l}73943 \text { elements (4300 linear hexa- } \\
\text { hedral C3D8R, 843 linear hexahedral } \\
\text { C3D8 and 68800 linear hexahedral } \\
\text { EC3D8R) }\end{array}$ \\
\hline Number of nodes & 81342 \\
\hline $\begin{array}{l}\text { Stable time } \\
\text { increment }\end{array}$ & $1 \cdot 10^{-7}-1 \cdot 10^{-9} \mathrm{~s}$ \\
\hline Computation time & 12 hours \\
\hline & \\
\hline
\end{tabular}

$$
\begin{aligned}
& u=u_{0}+\left(u_{1}-u_{0}\right) \xi^{3}\left(10-15+6 \xi^{2}\right) \\
& \text { for } t \in\left[t_{0}, t_{1}\right] \text { where } t \in\left[t_{0}, t_{1}\right]
\end{aligned}
$$

where: $u_{0}$ - initial punch position,

$u_{1}-$ final punch drive.

For numerical simulations, $\xi=\frac{t-t_{0}}{t_{1}-t_{0}}$ and $u_{0}=0$.

The smooth punch movement used in this analysis from to is presented in Figure 6.

The contact between the Eulerian and Lagrangian materials is enforced with the use of a general contact based on the penalty method which is less stringent than other procedures and allows a small penetration of the Eulerian material into the Lagrangian one. Seeds are generated on the Lagrangian element and anchor points are created on the Eulerian material surface. The 


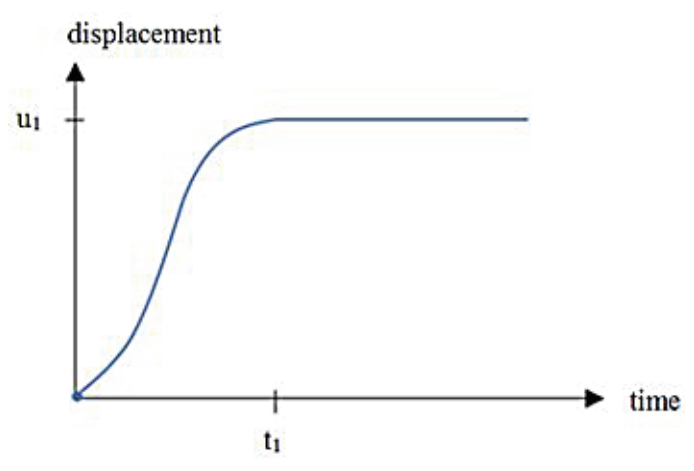

Figure 6. The smooth step amplitude definition with two data points

contact force between the seeds and anchor points is proportional to the penetration distance (Eq. 13) $[17,28]$ :

$$
F_{p}=k_{p} \cdot d_{p}
$$

where: $F_{p}-$ contact force,

$d_{p}^{p}$ - penetration distance,

$k_{p}^{p}$ - penalty stiffness depending on the Lagrangian and Eulerian material properties.

\section{RESULTS AND DISCUSSION}

In the numerical simulations of the KOBO extrusion process, the stress and strain distributions in the ingot, as well as the relationship between the punch force and the punch displacement, are found. An exemplary distribution of the effective plastic strain is shown in Figure $7 \mathrm{a}$ and $7 \mathrm{~b}$. The shape of the plastic zones confirms the results of experiments - see Figure. 8. One can also see that the plastic strain zone has a characteristic shape, which was confirmed in the research carried out by Bochniak et al. [5] (Figure 9).

Plots of the punch force vs. punch displacements obtained for two different die-processed material contact formulations considering the

a)
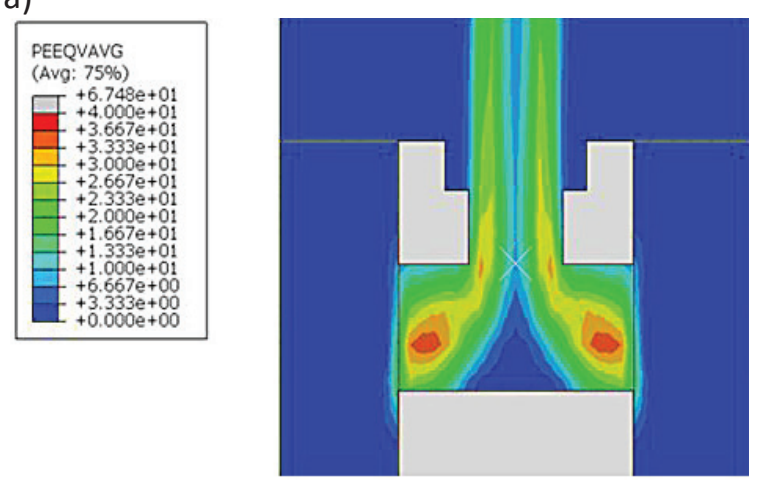

sliding on the die (KOBO-slip) and preventing sliding (KOBO-no slip) are presented in Figure 10 . The scattered results from the explicit procedure are smoothed with the use of spline filter. In the case of KOBO-no slip analysis, the rough contact between die and material is assumed.

As seen in Figure 10, the extrusion force in the KOBO process increases until it reaches the maximum when the plastic flow starts. The growth of the force is a result of the upsetting of a charge. However, in contrast to the conventional extrusion, the whole charge is not upset and therefore, the friction area and the friction force do not change significantly [5]. Afterwards, the extrusion force decreases due to the shortened contact area between the charge and the container. Although the force distributions are similar both in conventional and $\mathrm{KOBO}$ extrusion processes, they are on a different level. According to [3], the $\mathrm{KOBO}$ process is characterized by about half the force of the conventional extrusion.

The distributions of the extrusion force for the sliding on a die and without it have similar shape. After reaching the maximum punch force, the force in the no sliding case is about $20-25 \%$ lower than the one in the simulation where the sliding is allowed.

\section{THE MATERIAL MODEL}

In SPD problems including the KOBO extrusion process, the classical material processing methods are accelerated by the application of the additional cyclic load. Very good knowledge of the material properties, including the hardening characteristic and their variation due to the cyclic load, is very important in the analysis of large deformation problems. In this research, the Chaboche-Lemaitre (C-L) and Voce models describing

b)
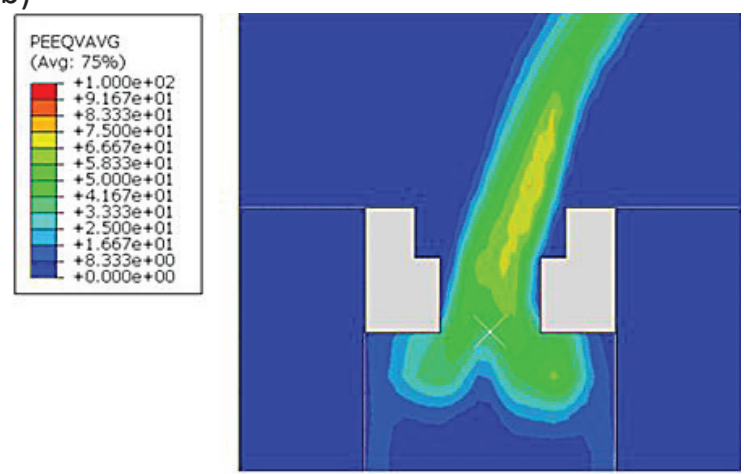

Figure 7. The effective plastic strain: (a) model with sliding on the die; (b) model without sliding on the die 


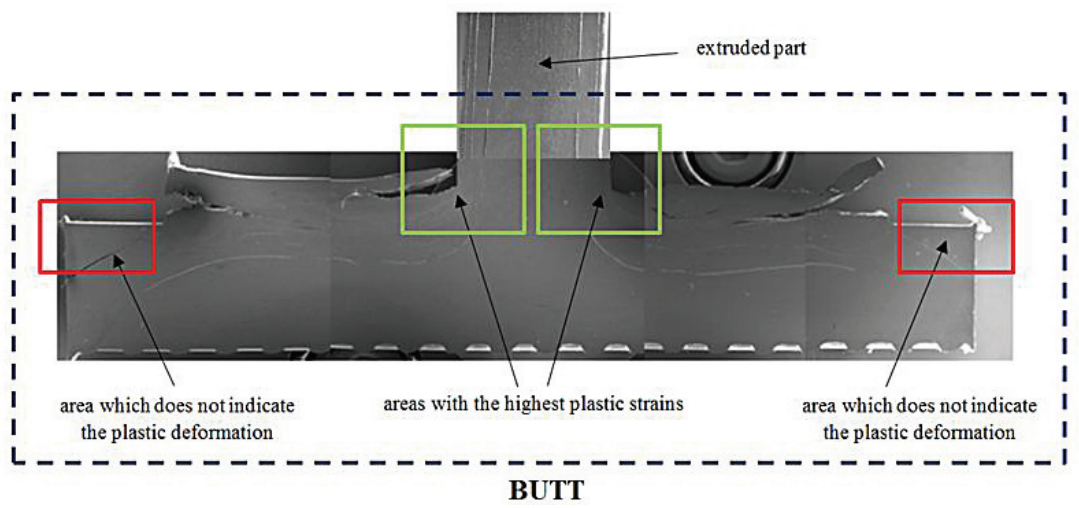

Figure 8. The microstructure of a butt - experimental research (results provided by M. Zwolak)

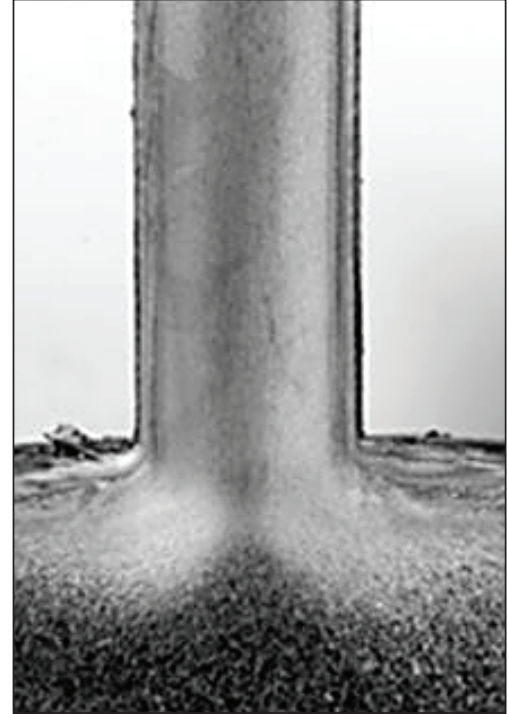

Figure 9. Longitudinal section of a copper wire along with butt extruded by KOBO method with a strain zone [5]

both non-linear kinematic and isotropic hardenings were considered. The kinematic hardening rule for the C-L model includes several components and is described as follows (Eq. 14) [29]:

$$
d \boldsymbol{x}_{i}=\frac{2}{3} c_{i} d \boldsymbol{\varepsilon}^{p}-\gamma_{i} \boldsymbol{x}_{i} d p
$$

where: $d \boldsymbol{x}_{\boldsymbol{i}}-i$-th backstress increment,

$d \varepsilon^{p}$ - plastic strain increment,

$d p$ - effective plastic strain increment,

$c_{i}-i$-th kinematic hardening parameter representing the translation rate of the yield surface,

$\gamma_{i}-i$-th kinematic parameter determining the relaxation rate of the yield surface translation as the plastic deformation accumulates.

The superposition of kinematic rules $\left(d \boldsymbol{x}_{\boldsymbol{i}}\right)$ for the C-L kinematic hardening model is the following (Eq. 15):

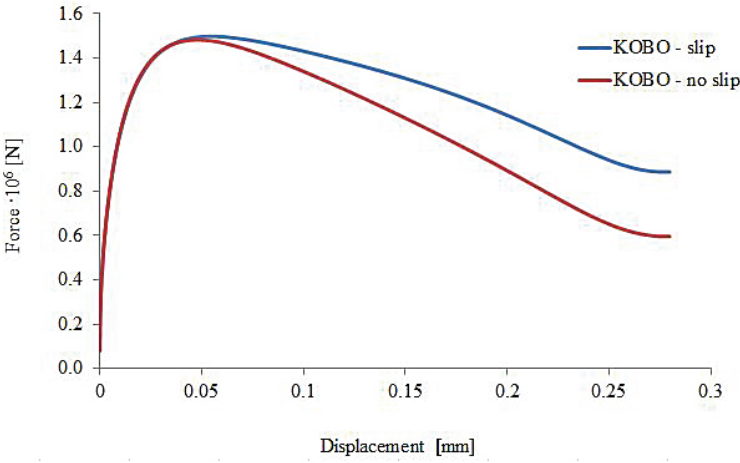

Figure 10. The relationship between the extrusion force and the punch displacement

$$
d \boldsymbol{x}=\sum_{i=1}^{n} d \boldsymbol{x}_{i}
$$

The C-L model with three backstresses $(n=3)$ was used in this paper for simulations of the KOBO extrusion process.

The isotropic hardening assuming the development of the plastic surface in the stress surface was described using the Voce model (Eq. 16) [30]:

$$
d r(p)=b(Q-r) d p
$$

where: $d r(p)$ - evolution of isotropic hardening, $Q$ - saturated value of the isotropic hardening component,

$b$-rate in which the saturation is achieved.

Using the C-L and Voce models, the behavior of the material is described by eleven material constants: six constants describing the kinematic hardening $\left(c_{i}, \gamma_{i}\right)$, two values which describe the isotropic hardening and two elastic constants Young modulus and Poisson's ratio $(v)$ and finally the yield stress $\left(\sigma_{y}\right)$.

The hardening parameters of a material can be obtained from symmetrical strain-controlled cyclic tension-compression tests. The methodology hardening parameters determination from cyclic 
loading tests was described in the author's previous works [31-33]. It allows achieving a good agreement between experimental and numerically generated stress-strain curves for cyclic loading tests.

The material hardening parameters for copper were determined using the experimental data from uniaxial cyclic loading test at the strain range $\Delta \varepsilon=$ $1.5 \%$ carried out by Annand [34]. The ChabocheLemaitre model with three backstresses was calibrated using the last stabilized hysteresis cycle procedure where the saturation of the isotropic hardening component takes place. The data from the half of the last cycle was used for the determination of six kinematic hardening parameters (Figure 11).

The plastic strain necessary for the determination of kinematic hardening parameters by the last stabilized cycle procedure was calculated using the additive decomposition of generalized strain assuming that the each pair of data $\left(\sigma_{i}, \varepsilon_{i}^{p}\right)$ should be specified with the strain shifted to $\varepsilon_{0}^{p}$ (Eq. 17):

$$
\varepsilon_{i}^{p}=\varepsilon_{i}-\frac{\sigma_{i}}{E}-\varepsilon_{0}^{p}
$$

where: $\varepsilon_{0}^{p}$ - translation of the yield surface,

$\varepsilon_{i}^{p}-$ plastic strain values on the stressstrain curve,

$\sigma_{i}-$ stress values on the stress-strain curve.

The isotropic hardening component which defines the evolution of the plastic surface in the stress space, was assessed as a half of the difference between the yield stress in tension and compression. The parameter $b$ determines the rate of the yield stress increase depending on the number of cycles up to the saturation. For low values of $b$, the material stabilizes relatively slowly, while high values of $b$ stabilize the isotropic hardening rapidly. The isotropic hardening for copper stabilizes in a few cycles and therefore, the high value of parameter was assumed $(b=17)$.

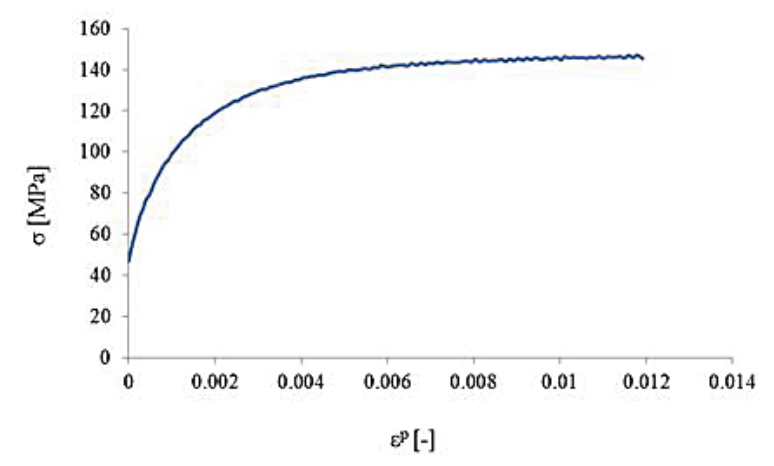

Figure 11. The last half cycle of the stress-plastic strain curve used for the calibration of the kinematic hardening parameters (experimental data from [34])
For the selection of the most reliable values of hardening parameters, the novel procedure based on the fuzzy logic was applied. The main advantage of this method is that the fuzzy logic approach considers some uncertainty of the input (hardening parameters) and output (error norm of numerical approximation of experimental stress-strain curves) data. The fuzzy analysis also includes the influence of the mapping model on the output variable(s) which is not taken into consideration in the classical statistic. The fuzzy logic method is particularly recommended for the tests which might not be carried out many times due to high costs, e.g. crash tests. Using the fuzzy set theory, on the basis of the expert knowledge, the statics might be built on the limited amount of experimental data. More information about the fuzzy logic theory is available in [35-37].

In this research, the input fuzzy variables are material hardening parameters, while the fuzzy output variable is the error norm $\|B\|$ which is calculated using the following formula (Eq. 18):

$$
\|B\|=\sqrt{\int_{\varepsilon}\left(\sigma_{\text {exp }}-\sigma_{a p p r}\left(c_{i}, \gamma_{i}, b, Q\right)\right)^{2} d \varepsilon}
$$

where: $\sigma_{\text {exp }}$-experimental stress values,

$$
\sigma_{\text {appr }} \text {-approximated stress values. }
$$

In the defuzzification step, the discrete value of the error norm $\|B\|$ is found. The hardening parameters, for which the discrete error norm was the smallest, were assumed to be optimal. This unique procedure of the determination of hardening parameters using the fuzzy logic was described in details in [31-33].

The kinematic and isotropic hardening parameters for copper, determined using the last stabilized cycle procedure and the fuzzy logic approach are contained in Table 3. The comparison of experimental and numerically generated stressstrain curves is shown in Figure 12a and 12b.

In the numerical simulations of the KOBO extrusion process, two phenomena which are very sensitive to the hardening parameters, namely ratcheting and mean stress relaxation, should be considered. During the cyclic loading, most materials exhibit the mean stress relaxation, as well as the strain ratcheting. Both are characterized by unclosed hysteresis loops and stabilization reached after certain number of loading cycles.

The ratcheting is the directional progressive accumulation of plastic deformation in a material under the non-symmetrical stress-controlled cyclic 
Table 3. Material hardening parameters for cooper used in this research

\begin{tabular}{|c|c|c|}
\hline \multicolumn{2}{|c|}{ Value (the last stabilized cycle procedure } & Value (the fuzzy logic analysis) \\
\hline \multicolumn{3}{|c|}{ Isotropic hardening } \\
\hline$Q[\mathrm{MPa}]$ & 148.0 & 149.4 \\
\hline$b[-]$ & 17.0 & 16.5 \\
\hline \multicolumn{3}{|c|}{ Kinematic hardening } \\
\hline$c_{1}[\mathrm{MPa}]$ & 28253.0 & 26954.2 \\
\hline$c_{2}[\mathrm{MPa}]$ & 23918.0 & 27366.9 \\
\hline$c_{3}[\mathrm{MPa}]$ & 7305.0 & 8154.5 \\
\hline$\gamma_{1}[-]$ & 5712.8 & 5459.7 \\
\hline$\gamma_{2}[-]$ & 959.6 & 778.4 \\
\hline$\gamma_{3}[-]$ & 346.4 & 380.8 \\
\hline
\end{tabular}

a)

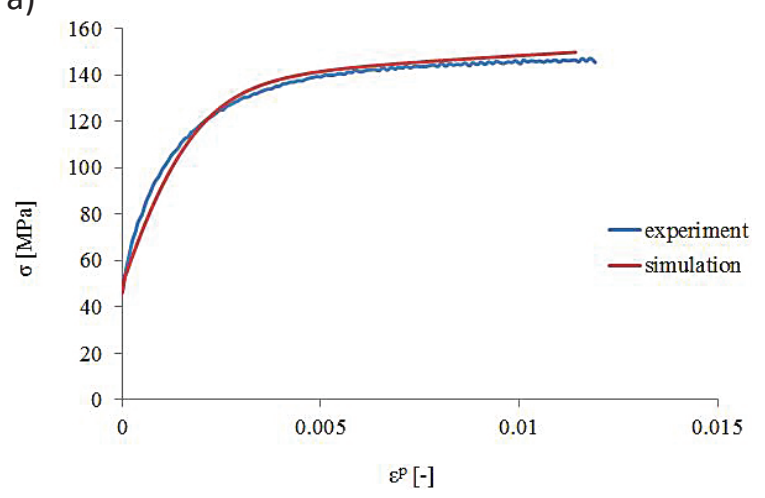

b)

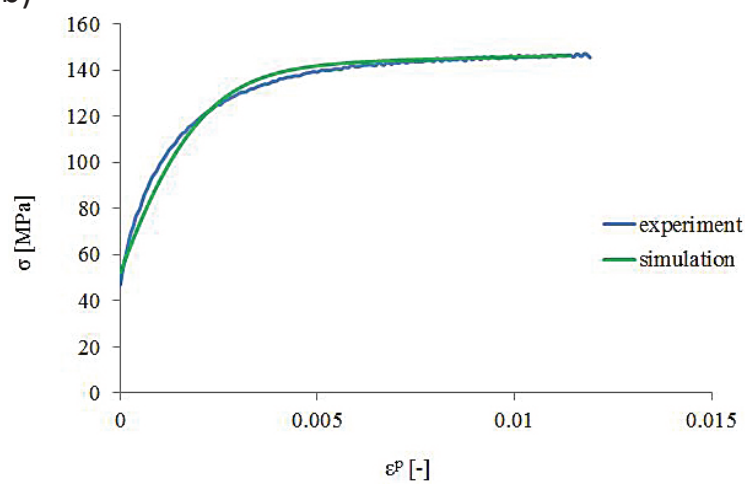

Figure 12. The last half cycle of the stress-strain curve used for the calibration of the kinematic hardening parameters: (a) the last stabilized cycle procedure; (b) the fuzzy logic approach (experimental data from [34])

loading with a non-zero mean stress $[38,39]$. This phenomenon was extensively examined and described in [40-43]. One can see that large $Q$ isotropic hardening component causes the gradual deceleration, and even blocking the ratcheting progress [44].

The numerical simulation of the ratcheting for copper in 1D case is shown in Figure 13. The load history applied in a test is shown in Figure 14. The stabilization of hysteresis curves is the result of an isotropic hardening. Further loading cycles can cause very small strain increments - material response is almost only elastic. In the KOBO extrusion process, large isotropic hardening should be avoided because it will block the extrusion processing. The knowledge about the material hardening characteristic is essential for the optimization of the KOBO process and in terms of the frequency of the die oscillations.

On the other hand, the non-symmetrical load can help to reduce the mean stress and in this way decrease the force applied to the stamp. The mean stress relaxation results in the vertical shift of the

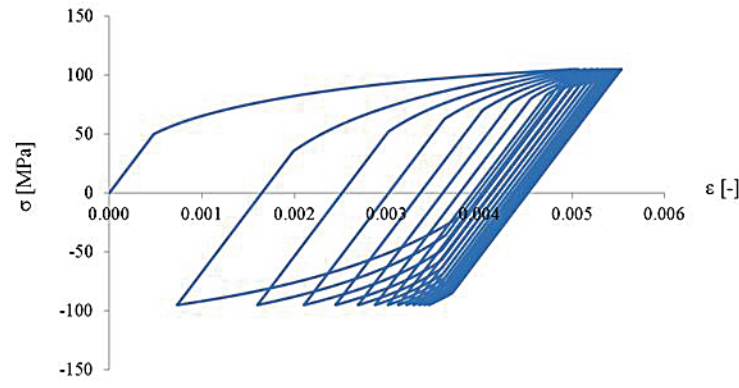

Figure 13. The ratchetting for copper used in this research (numerical simulation)

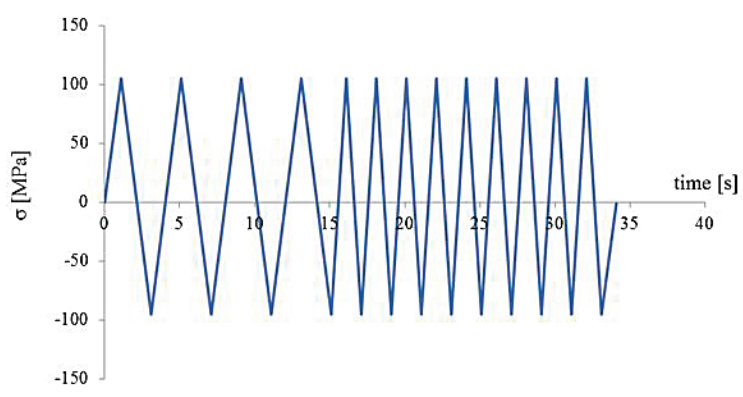

Figure 14. The load vs. time for the ratcheting 
stress-strain curves towards the zero-mean stress position for a constant strain amplitude $[45,46]$. The material response for non-symmetrical straincontrolled cyclic loading is also very sensitive to the material hardening parameters. Depending on the material hardening parameters (especially isotropic components), the plastic shakedown might occur after certain number of cycles. The theoretical information about the mean stress relaxation is contained in [47-49].

The change of strain in time for the mean stress relaxation test is shown in Figure 15. Numerical simulation of the mean stress relaxation for copper subjected to non-symmetrical load for 1D case is presented in Figures 16 and 17. The increase of the plastic surface and the stabilization of stressstrain curves after several loading cycles were observed. The growth of the yield locus is caused by the isotropic hardening which dominates over the kinematic components. The mean stress decreases from approximately $8 \mathrm{MPa}$ to $0.9 \mathrm{MPa}$ (see Figure 17). The decrease of the mean stress is rapid during the early cycles of loading and afterwards, the mean stress has a near constant value.

Considering the KOBO extrusion process, the decrease of the mean stress during loading cycles is essential for its normal course. However, the mean stress relaxation test should be analyzed in

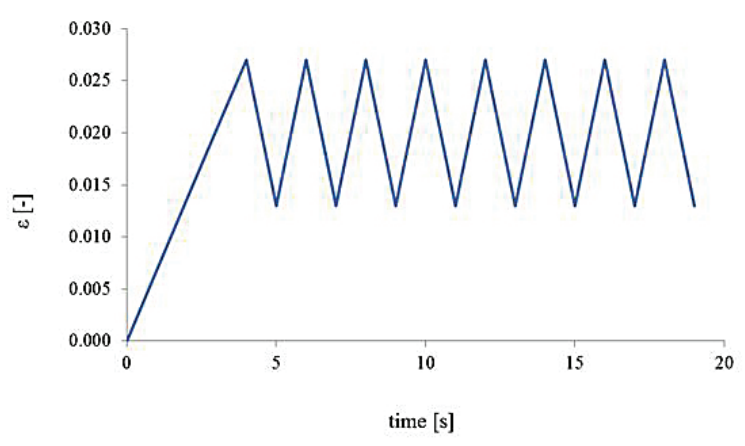

Figure 15. The strain vs. time for the mean stress relaxation test

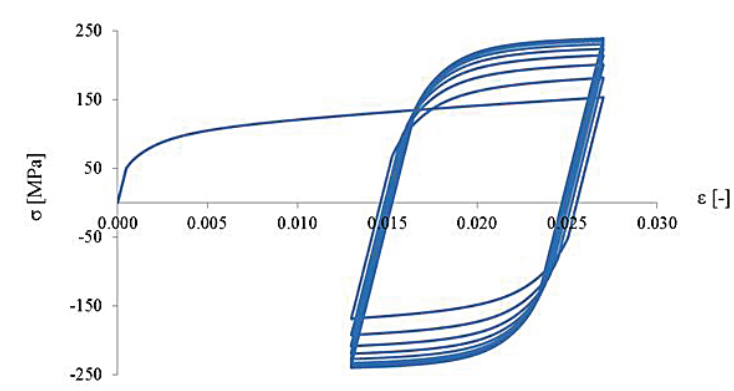

Figure 16. The mean stress relaxation for copper used in this research (numerical simulation)

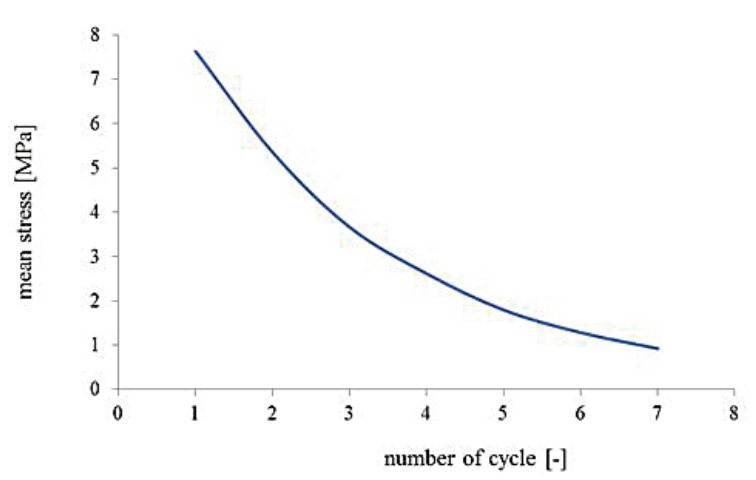

Figure 17. The mean stress vs. time (numerical simulation)

conjunction with the ratcheting phenomena. For optimization of the KOBO process, the achievement of the decrease of a mean stress while avoiding the quasi-shakedown response of the material during the ratcheting is essential and constitutes a challenge for both experimental and numerical studies.

\section{SUMMARY AND CONCLUSIONS}

Numerical simulation of the KOBO extrusion process was presented in this paper. The Eulerian-Lagrangian (CEL) method was used in computer computations. The problem was solved as $3 \mathrm{D}$ dynamic explicit integration procedure. The main advantages of CEL method are: no need of remeshing and stability even for complicated shapes. In the CEL analysis, the deformed Lagrangian mesh moves through the Eulerian fixed mesh. The volume of material which goes through the Eulerian mesh is calculated and then the Lagrangian solution variables (e.g. stress, mass, energy) are matched to the Eulerian solution. However, the explicit integration is characterized by very short stable time increments and usually millions of load increments are required. For this reason, the computations might last for a long time even on powerful workstations.

Many numerical simulations of the KOBO extrusion process have been already done within this research. The results show the characteristic shape of the plastic zones which were verified in experimental tests. The obtained results show that the highest plastic strain occurs in the middle of the butt.

The distribution of the extrusion force versus the punch displacement is similar to the conventional extrusion process. However, the stamp force has a significantly lower level. It can be seen that 
the model which does not include the sliding on the matrix is characterized by the lower extrusion force in the second stage of the KOBO extrusion process. It is caused by better material processing.

Numerical simulations also show that the influence of hardening parameters of extruded material on the KOBO process is essential for its proper course. It was noted that the isotropic hardening components can cause unwanted stabilization of the ratcheting phenomena. On the other hand, the decrease of a mean stress during the relaxation is beneficial for the KOBO extrusion process. However, such determination of hardening parameters which ensures the decrease of the mean stress in conjunction with avoiding the quasi-shakedown material response is essential for the proper course of the process.

The research contained in this paper shows the numerical simulations that enable to optimize the process and select the optimal shape of a die as well as the amount of die oscillations in order to decrease its wearing. So far, only the cold extrusion process has been analyzed. The further research will focus on the including the thermo-mechanical aspects of this process resulting from the friction between the processed material and tools, as well as resulting from the material plastic deformations.

\section{REFERENCES}

1. Sun Y.D., Chen Q.R. Numerical simulation of extrusion process and die structure optimization for a complex magnesium doorframe. The International Journal of Advanced Manufacturing Technology, 80, 2015, 495-506.

2. Łyszkowski R., Łazińska M., Zasada D. The Influence of A Cross-Channel Extrusion Process on The Microstructure and Properties of Copper. Metals, 12, 2019, 3995.

3. Bochniak W., Korbel A., Ostachowski P., Ziółkiewicz S., Borowski J. Wyciskanie metali i stopów metodą KOBO. Obróbka Plastyczna Metali, 24(2), 2013, 83-97.

4. Pawłowska B., Śliwa R.E., Zwolak M. Posibilities to obtain products from 2024 and 7075 chips in the process of consolidation by KOBO extrusion. Archives of Metallurgy and Materials, 64, 2019, 1213-1221.

5. Bochniak W., Korbel A. Lüders deformation and superplastic flow of metals extruded by KOBO method. Philosophical Magazine, 93, 2013, 1883-1913.

6. Rozwalka J., Płaczek G., Andrzejewski D. Wypływ procesu KOBO na właściwości stopu $\mathrm{Cu}-$ Cr1Zr. Archives of Metallurgy and Materials, 28(2), 2017, 107-122.
7. Arentoft M., Gronostajski Z., Niechajowicz A., Wanheim T. Physical and mathematical modelling of extrusion process. Journal of Materials Processing Technology, 106(1-3), 2000, 2-7.

8. Willams A.J., Croft T.N., Cross M. Computational modelling of metal extrusion and forging. Journal of Materials Processing Technology, 125-126, 2002, 573-582.

9. Ayer Ö., Özmen B.G., Karakaya I. An Optimization Study for Bridge Design of a Porthole Extrusion Die. Advances in Science and Technology Research Journal, 13(4), 2019, 270-275.

10. Winiarski G., Bulzak T.A., Wójcik Ł., Szala M. Effect of Tool Kinematics on Tube Flanging by Extrusion with a Moving Sleeve. Advances in Science and Technology Research Journal, 13(3), 2019, 210-216.

11. Kowalczyk-Gajewska K., Stupkiewicz S. Modelling of texture evolution in KOBO extrusion process. Archives of Metallurgy and Materials, 58(1), 2013, 113-118.

12. Gusak A., Danielewski M., Korbel A., Bochniak M., Storozhuk N. Elementary model of severe plastic deformation by KoBo process. Journal of Applied Physics, 115, 2014, 034905.

13. Qui G., Henke S., Grabe J. Application of a Coupled Eulerian-Lagrangian approach on geomechanical problems involving large deformation. Computers and Geotechnics, 38(1), 2011, 30-39.

14. Cremonesi M., Franci A., Idelsohn S., Oñate E. A state of the Art Review of the Particle Finite Element Method (PFEM). Archives of Computational Methods in Engineering, 27, 2020, 1709-1735.

15. Ryzińska G., Śliwa R. Ductile fracture phenomenon during extrusion of bimetal rods. Archives of Metallurgy and Materials, 51(1), 2006, 109-118.

16. Ryzińska G., Śliwa R. The theoretical and experimental researches of $\mathrm{Pb}-\mathrm{Al}$ composite materials extrusion. Metalurgija, 51(3), 2012, 293-297.

17. Qiu G., Henke S., Grabe J. Applications of Coupled Eulerian-Lagrangian Method to Geotechnical Problems with Large Deformations. Available online: http://www.simulia.com/download/pdf2009/ Qiu_SCC2009.pdf (access: 10 October 2020).

18. Després B. Numerical Methods for Eulerian and Lagrangian Conservation Laws. Springer, 2017.

19. Alizadeh A., Jafarmadar S., Zekri H.J. Use of One and Two Horizontal Plates to Reduce the Drag Force on the Rigid Cylinder Located Inside the Channel: Approach of the Immersed Interface Method. Advances in Science and Technology Research Journal,, 13(4), 2019, 188-193.

20. Ryzińska G., Skrzat A., Śliwa R. Sprzężona analiza Eulera-Lagrange'a w modelowaniu procesu wyciskania. Obróbka Plastyczna Metali, 26(1), 2015, 73-92.

21. Ko J., Jeong S., Kim J. Application of a Coupled Eulerian-Lagrangian Technique on Constructabil- 
ity Problems of Site on Very Soft Soil. Applied Sciences, 7, 2017, 1080.

22. Ducobu F., Arrazola P.J., Rivière-Lorphèvre E., Ortiz deZarate G., Madariaga A., Filippi E. The CEL Method as an Alternative to the Current Modelling Approaches for Ti6A14V Orthogonal Cutting Simulation. Procedia CIRP, 58, 2017, 245-250.

23. Skrzat A. Application of Coupled Eulerian-Lagrangian Approach in Metal Forming Simulations. Zeszyty Naukowe Politechniki Rzeszowskiej seria Mechanika, 84(4/12), 2012, 25-35.

24. Benson D.J.A multi-material Eulerian formulation for the efficient solution of impact and penetration problems. Computational Mechanics, 15, 1995, 558-571.

25. Benson D.J., Okazawa S. Contact in a multi-material Eulerian finite element formulation. Computer Methods in Applied Mechanics and Engineering, 193, 2004, 4277-4298.

26. Bakroon M., Aubram D., Rackwitz F. Geotechnical Large Deformation Numerical Analysis Using Implicit and Explicit Integration. Proc. of $3^{\text {rd }}$ International Conference on New Advances in Civil Engineering, Helsinki, Finland 2017, 26-36.

27. Söderlind G. Numerical Methods for Different Equations. An Introduction to Scientific Computing. Springer, 2017.

28. Sherburn J., Hammons M.I., Roth M.J. Modeling finite thickness slab perforation using a coupled Eulerian-Lagrangian approach. International Journal of Solids and Structures, 51(25-26), 2014, 4406-4413.

29. Dunne F., Petrinic N. Introduction to Computational Plasticity. Oxford University Press, 2005.

30. Wójcik M., Skrzat A. The application of Chaboche model in uniaxial ratcheting simulations. Advances in Manufacturing Science and Technology, 44(2), 2020, 57-61.

31. Skrzat A., Wójcik M., Bąk Ł. Fuzzy logic enhancement of material strain hardening data obtained in the Heyer's test. Archives of Metallurgy and Materials, 64(3), 2019, 1059-1065.

32. Wójcik M., Skrzat A. Fuzzy logic enhancement of material hardening parameters obtained from tension-compression test. Continuum Mechanics and Thermodynamics, 32, 2020, 959-969.

33. Wójcik M., Skrzat A. Identification of ChabocheLemaitre combined isotropic-kinematic hardening model parameters assisted by the fuzzy logic analysis. Acta Mechanica (in press).

34. https://classes.engineering.wustl.edu/2009/spring/ mase5513/abaqus/docs/v6.6/books/bmk/default.htm ?startat $=$ ch03s02ach162.html (access: 29.11.2020).

35. Möller B., Beer M. Fuzzy Randomness. Uncertainty in Civil Engineering and Computational Mechanics. Springer, 2004.
36. Skrzat A., Wójcik M. The application of fuzzy logic in engineering applications. ZN Mechanika, 298(90/4), 2018, 505-518.

37. Bolotin A. Quantum mechanical approach to fuzzy logic modelling. Mathematical and Computer Modelling, 34(7-8), 2001, 937-945.

38. Paul S.K., Sivaprasad S., Dhar S., Tarafder S. True stress control asymmetric cyclic plastic behavior in SA333 C-Mn steel. International Journal of Pressure and Pipping, 87, 2010, 440-446.

39. Paul S.K. A critical review of experimental aspects in ratcheting fatigue: microstructure to specimen to component. Journal of Materials Research and Technology, 8(5), 2019, 4894-4914.

40. Xu L.-Y., Fan J.-S., Yang Y., Wang C. Ratcheting assessment of low yield point steel BLY160: Experimental analysis and constitutive modelling. Mechanics of Materials, 148, 2020, 103460.

41. Li H., Kang G., Yu C. Modeling uniaxial ratchetting of magnesium alloys by a new crystal plasticity considering dislocation slipping, twinnung and detwinning mechanisms. International Journal of Mechanical Sciences, 179, 2020, 105660.

42. Karolczuk A. Ratcheting simulation in a titanium-steel bimetallic plate based on the Chaboche hardening model. Acta Mechanica et Automatica, 10(4), 2016, 265-270.

43. Tian J., Yang Y., Zhang L.-P., Shao X.-J., Du J., Kan Q.-H. Ratcheting Behavior of SA508-3 Steel at Elevated Temperature: Experimental Observation and Simulation. Acta Metallurgica Sinica (English Letters), 30, 2017, 822-828.

44. Skrzat A., Wójcik M. An identification of the material hardening parameters for cyclic loading - experimental and numerical studies. Archives of Metallurgy and Materials, 65(2), 2020, 779-786.

45. Wang C.H., Rose L.R.F. Transient and steady-state deformation at notch root under cyclic loading. Mechanics of Materials, 30(3), 1998, 229-241.

46. Hu W., Wang C.H., Barter S. Analysis of Cyclic Mean Stress Relaxation and Strain Ratchetting Behaviour of Aluminium 7050. DSTO Aeronautical and Maritime Research Laboratory, 1999.

47. Shit J., Dhar S., Acharyya S. Experimental and numerical simulation of carbon manganese steel for cyclic plastic behaviour. International Journal of Engineering, Science and Technology, 2(4), 2010, 71-84.

48. Wallbrink C., Hu W. A Strain-Life Module for CGAP: Theory, User Guide and Examples. DSTO Defence Science and Technology Organisation, 2010.

49. Paul S.K., Stanford N., Taylor A., Hilditch T. The effect of low cycle fatigue, ratcheting and mean stress relaxation on stress-strain response and microstructural development in a dual phase steel. International Journal of Fatigue, 80, 2015, 341-348. 